\title{
Niveles de ira en practicantes de boxeo y kickboxing: diferencias en función de la disciplina y el nivel deportivo
}

\section{Anger intensity in boxing and kickboxing practitioners: differences based on discipline and competitive level}

\author{
Niveis do ira na prática e kickboxing boxe: diferenças \\ em termos de disciplina e nível esportivo
}

\author{
José Ignacio Menéndez Santurio y Javier Fernández-Río
}

Universidad de Oviedo, Facultad de Formación del Profesorado y Educación.

Resumen: El objetivo fundamental de este trabajo fue estudiar el perfil de ira desde una perspectiva multidimensional en deportes de contacto pleno (al K.O.). En el estudio participaron 269 hombres entre 16 y 39 ańos divididos en 5 grupos por niveles deportivos: no practicantes, practicantes regulares, competidores regionales, nacionales e internacionales. Se les administró un cuestionario sociodemográfico y el Inventario de la Expresión de la Ira Estado-Rasgo (STAXI-2). Se hallaron diferencias significativas $(p<, 05)$ en función del nivel deportivo en todas las escalas y subescalas del inventario exceptuando en la expresión y control interno de ira. Las pruebas post hoc revelaron cómo el grupo de deportistas internacionales presentó niveles significativamente más bajos de ira que los practicantes regulares y los no practicantes. Asimismo, se han establecido correlaciones significativas entre la edad, los años, los días y las horas de práctica y diferentes dimensiones de la ira. No se han encontrado diferencias significativas en el nivel de ira entre los practicantes regulares de estas disciplinas y los no practicantes de actividad física. Finalmente, tampoco se han encontrado diferencias significativas en los niveles de ira entre los practicantes de boxeo y los de kickboxing. Se discuten los hallazgos del estudio, se exponen las limitaciones encontradas, futuras líneas de investigación y aplicaciones prácticas.

Palabras clave: deportes de contacto, emoción, deportes de combate, artes marciales.

Abstract: The main goal of this study was to assess the anger profile of direct-contact (K.O.) sports' sportsmen from a multidimensional perspective. A total of 269 men, ages between 16 and 39 years old, agreed to participate. They completed the State-Trait Anger Expression Inventory (STAXI-2). Participants were placed in 5 groups based on their competitive level: no-practice, regular practice, state competitors, national competitors, and international competitors. Significant differences $(p<, 05)$ were found among groups in the different scales and subscales of the inventory, except expression and internal control of anger. Post hoc analyses revealed that the international competitors showed significant lower anger levels than no-practice and regular practice participants. Significant correlations were found among variables such as age, years, days, hours of practice and different dimensions of anger. No significant differences were found between no-practice and regular practice participants' anger level. Finally, no significant differences were found between boxing and kickboxing participants' anger levels, either. Findings are discussed, limitations are exposed, future lines of research are introduced, and practical applications are included. Keywords: contact sports, emotion, combat sports, martial arts.

Resumo: O objetivo fundamental deste trabalho foi estudar o perfil da ira a partir de uma perspetiva multidimensional em desportos de contacto pleno (ao K.O.). No estudo participaram 269 homens entre os 16 e os 39 anos, divididos em 5 grupos por níveis desportivos: não praticantes, praticantes regulares, competidores regionais, nacionais e internacionais. Foi-lhes aplicado um questionário sociodemográfico e o Inventário da Expressão da Ira Estado-Traço (STAXI-2). Foram encontradas diferenças significativas $(\mathrm{p}<, 05)$ em função do nível desportivo em todas as escalas e subescalas do inventário, exceto na expressão e controlo interno da ira. Os post hoc revelaram que o grupo de desportistas internacionais apresentou níveis significativamente mais baixos de ira do que os praticantes regulares e os não praticantes. Além disso, foram estabelecidas correlaçôes significativas entre a idade, os anos, os dias e as horas de prática e diferentes dimensóes da ira. Não foram encontradas diferenças significativas no nível de ira entre os praticantes regulares destas modalidades e os não praticantes de atividade física. Finalmente, também não foram encontradas diferenças significativas nos níveis de ira entre os praticantes de boxe e os de kickboxing. São debatidos os resultados do estudo, e expostas as limitaçôes encontradas, futuras linhas de investigação e aplicaçóes práticas. Palavras-chave: desportos de contacto, emoção, desportos de combate, artes marciais.

\section{Introducción}

La práctica de artes marciales y deportes de combate (AAMM y DDCC) ha crecido significativamente en los últimos ańos

Dirección para correspondencia [Correspondence address]: José Ignacio Menéndez Santurio. Universidad de Oviedo. Departamento de Ciencias de la Educación. Facultad de Formación del Profesorado y Educación. C/ Aniceto Sela, s/n. Despacho 219. 33005, Oviedo (España). E-mail: UO194643@uniovi.es (van Bottenburg, Rijnen y Sterkenburg, 2005; Loftian, Ziaee, Amini y Mansournia, 2011). Esto ha llevado a un creciente interés por acercase al campo de estudio de estas modalidades deportivas por parte de investigadores de todo el mundo (Vertonghen y Theeboom, 2010). Los beneficios psicológicos de su práctica han sido muy discutidos a lo largo de los ańos (Vertonghen y Theeboom, 2010), a pesar de que diferentes 
investigaciones han reportado tanto resultados negativos (Reynes y Lorant, 2002) como positivos (McDiarmid, 2007; Theeboom y De Knop, 1999).

Las variables psicológicas que se han estudiado dentro del campo de las AAMM y DDCC han sido muy diversas: ansiedad (Marks, Bukowska y Biéc, 2012), inteligencia emocional (Szabo y Urbán, 2014), resolución de conflictos (Björkqvist y Varhama, 2001), hostilidad (Daniels y Thornton, 1990) y especialmente, agresividad (Adler, 2003; Ali, Emrah, Fahri, Necmettin y Gülcan, 2010; Endresen y Olweus, 2005). La ira también ha sido otra variable objeto de análisis (Foster, 1997; Oliva y Calleja, 2010; Steyn y Roux, 2009) aunque tradicionalmente se ha estudiado a partir del constructo irahostilidad-agresión sin atender a la multidimensionalidad de esta emoción (Reynes y Lorant, 2001, 2002, 2004). En los últimos años ha crecido el número de trabajos que han explorado esta variable desde un prisma multidimensional, tanto en el ámbito de las AAMM y DDCC como en el del deporte en general (Bolgar, Janelle y Giaccobbi, 2008; Dunn, Gotwals, Causgrove, Dunn y Syrotuik, 2006; Oliva y Hernández, 2009; Oliva, Calleja y Hernández, 2011, 2012; Robazza, Bertollo y Bortoli, 2006; Ruiz y Hanin, 2011).

El término ira se ha empleado de forma intercambiable con los términos hostilidad y agresión debido, principalmente, a la ambigüedad de sus definiciones y al fuerte solapamiento entre ellas (Carrasco y González, 2006). Esto ha llevado a muchos a incluirlos bajo un continuo que denominó Síndrome AHA: Anger (ira), Hostility (hostilidad), Aggression (agresión) (Spielberger, Jacobs, Russell y Crane, 1983; Spielberger et al., 1985). Sin embargo, los tres conceptos pertenecen a constructos diferenciados. La ira es un componente emocional constituido por sentimientos de diferentes intensidades, desde el enfado a la irritación o la furia, que suele acompañarse de activación fisiológica, neuroquímica, experiencia subjetiva de sentimientos y un determinado modo de expresión y afrontamiento (Spielberger et al., 1985). La hostilidad es una actitud negativa hacia una o más personas que es reflejada en un juicio decididamente desfavorable del blanco (Berkowitz, 1996) y que generalmente motiva actos agresivos de destrucción y daño físico de objetos y/o personas (Spielberger et al., 1983, 1985). Finalmente, la agresión es entendida como aquella conducta que tiene como objetivo principal herir física o psicológicamente a otro individuo (Berkowitz, 1996). Por lo tanto, podríamos decir que mientras que la ira representa el componente emocional y la hostilidad el papel cognitivoevaluativo, la agresión conforma el rol comportamental del continuo AHA.

Spielberger et al. (1985) afirman que la ira puede estudiarse a partir de dos manifestaciones: el estado de ira y el rasgo de ira. El estado de ira se definiría como la experiencia pasajera de sentimientos subjetivos de tensión, enfado, irritación o furia como respuesta a una serie de eventos y generalmen- te acompañada de aumentos en la activación fisiológica. Por otro lado, el rasgo de ira hace referencia a la disposición de experimentar episodios de ira de forma frecuente y/o pronunciada. Estos episodios de ira están causados por diferentes motivos: la percepción de un amplio rango de situaciones como provocadoras, la predisposición a experimentar situaciones negativas intensas o bien debido a la interacción de ambos factores (Spielberger et al., 1985).

El concepto de ira, además, implica dos dimensiones adicionales: la expresión y el control de ira. Según la conceptualización de Miguel-Tobal, Casado, Can-Vindel y Spielberger (2001), estas dimensiones están constituidas por cuatro componentes diferenciados: la expresión interna, la expresión externa, el control interno y el control externo. El primero de ellos hace referencia al tipo de ira en el que tras experimentar situaciones de irritación o furia, el individuo reprime esos sentimientos redirigiéndolos hacia el interior. En el segundo caso, por el contrario, la persona que experimenta los episodios de ira los expresa externamente hacia personas u objetos de su alrededor. En el tercer componente, la persona trata de suprimir esos sentimientos a través de la calma y el sosiego mientras que, finalmente, en el cuarto y último componente, el individuo trata de controlar los sentimientos de furia o enfado evitando su expresión hacia objetos o personas de su entorno.

El deporte es un medio donde la presencia de componentes como la ira juegan un papel fundamental para el correcto desempeño de la actividad (Proios, 2012). Quizá por ello, la gran mayoría de las investigaciones que se han realizado sobre esta variable dentro del ámbito de las AAMM y DDCC se han centrado en explorar esta cuestión (Ali, Ichraf, Khaled, Liwa y Ali; 2013; Brown et al., 1995; Chiodo et al., 2011; Davenport, 2006; McGowan y Miller, 1989; Oliva, Calleja y Hernández, 2011; Pieter y Pieter, 2008; Ruiz y Hanin, 2003, 2004a; 2004b; Szabo y Parkin, 2001). Han sido pocas investigaciones las que han tratado de estudiar la transferencia emocional de la práctica deportiva a la vida diaria en AAMM y DDCC, centrándose principalmente en el colectivo adolescente (Egan, 1993; McDiarmid, 2007; McGowan, Pierce y Jordan, 1991; Steyn y Roux, 2009: Vertonghen, Theeboom y Pieter, 2014; Ziaee, Loftian, Amini, Mansournia y Memani, 2012) y en el ámbito no competitivo (Focht, Bouchard y Murphey, 2000) y dejando excluidos los relacionados con el ámbito competitivo.

Boostani y Boostani (2012) realizaron una comparación sobre los niveles de agresión y sus componentes en deportistas de no contacto (natación), contacto controlado (karate), de contacto pleno (kickboxing) y un grupo control de no practicantes. Los resultados señalaron diferencias significativas en las variables de ira, hostilidad, agresividad física y verbal entre los practicantes de kickboxing y el resto de participantes. En este trabajo fue el grupo de kickboxing el que presentó 
mayores niveles en las variables citadas anteriormente. Sofía y Fernando (2010) analizaron la relación entre percepción de amenaza subyacente a la experiencia de estrés y ansiedad, ira y agresividad en deportes con diferentes grados de contacto físico (defensa personal, kickboxing y fútbol). Los resultados mostraron la correlación positiva entre ansiedad e ira y ansiedad y percepción de amenaza. Asimismo, se encontró que la ansiedad tiende a aumentar en deportes colectivos, mientras que la ira y la agresividad parecen decrecer. En este estudio, los practicantes de fútbol fueron los que presentaron mayores niveles de agresividad e ira, seguidos de los de kickboxing y defensa personal. van Ingen (2011) analizó un proyecto de boxeo recreativo destinado a mujeres y transexuales víctimas de violencia. Las entrevistas realizadas a las participantes señalaron la especial vinculación de la ira con la violencia, así como la utilidad del boxeo como deporte especialmente poderoso para reducir la ira por su efecto catártico.

Uno de los mayores beneficios del deporte es su transferencia: la capacidad de trasladar lo aprendido en el contexto deportivo a las situaciones cotidianas de nuestra vida. Danish, Nellen y Owens (1996) plantean la necesidad de utilizar el deporte como base para producir aprendizajes beneficiosos para el desarrollo psicosocial del individuo, más allá de la inmediatez de lo que ocurre y se aprende en el contexto deportivo. Hasta la fecha son casi inexistentes los trabajos que han tratado de explorar la ira en el contexto de la vida diaria con competidores de AAMM y DDCC. La casi totalidad de investigaciones han analizado las disciplinas donde el contacto no existe o es controlado (judo, taekwondo y karate principalmente), siendo escasos los trabajos relacionados con deportes como el boxeo y las modalidades de kickboxing de contacto pleno: K1 rules, low kick y full contact (WAKO, 2012). Los pocos estudios existentes sobre la ira en este tipo de disciplinas han estudiado esta emoción sin profundizar en las diferentes dimensiones que la conforman. No solo esto, sino que además, ninguno de ellos ha explorado la interacción de esta variable con otras como el tiempo de práctica o el nivel deportivo.

En base a todo lo anterior, nos planteamos los siguientes objetivos: 1) analizar si existen diferencias en los niveles de ira en función de la disciplina y el nivel deportivo en una muestra de deportistas de contacto pleno y un grupo de no practicantes y 2) determinar las posibles correlaciones existentes entre la ira y la edad, los años, los días y las horas semanales de práctica. En base a estos dos objetivos anteriormente expuestos se plantearon las siguientes hipótesis: 1) habrá diferencias significativas en las diferentes dimensiones de la ira en función del nivel deportivo, 2) los ańos de práctica, la edad, las horas y los días semanales de entrenamiento correlacionarán significativamente con las diferentes dimensiones de la ira, 3) no habrá diferencias significativas entre los no practicantes y los practicantes regulares de los dos deportes en las diferentes dimensiones de la ira y 4) no habrá diferencias significativas en las diferentes dimensiones de la ira en función del deporte.

\section{Método}

\section{Diseño y participantes}

Se realizó un estudio cuantitativo de tipo comparativo transversal que ha seguido un diseño transcultural de dos grupos diferenciados: deportistas y no deportistas (Ato, López y Benavente, 2013). En la investigación participaron 269 hombres de edades comprendidas entre los 16 y los 39 ańos $(M=26,5$; DT=5,97) divididos en 5 grupos por nivel: 1) no practicante (no practica actividad física de forma regular), 2) practicante regular (entrena en la modalidad que indica al menos 3 horas a la semana desde, como mínimo, un ańo), 3) competidor regional (practica regularmente y acude a competiciones de carácter regional), 4) competidor nacional (practica regularmente y forma parte de las selecciones autonómicas y/o compite profesionalmente a nivel nacional) y 5) competidor internacional (practica regularmente y forma parte de las selecciones nacionales y/o compite profesionalmente a nivel internacional). En relación a la disciplina deportiva, en el caso de ser multipracticante, los participantes tuvieron que seleccionar aquella modalidad por la que se sentían más identificados.

De los participantes deportistas, 108 eran practicantes activos de boxeo y 107 de kickboxing. De media practican la disciplina desde hace 7,50 ańos (DT=6,06) y entrenan 9,92 horas $(\mathrm{DT}=6,52)$ a lo largo de 4,50 días semanales (DT=1,28). Los 54 restantes pertenecían a población general no practicante $(\mathrm{M}=23,8, \mathrm{DT}=6,23)$. Este grupo procedía del entorno familiar, laboral y de ocio de los investigadores. El objetivo era conformar un grupo de no practicantes de actividad física lo más amplio y heterogéneo posible que pudiera representar a la población general y realizar comparaciones con los otros grupos. En la tabla 1 se presenta el número y edad de los participantes. Las medias de la tabla están referidas a su promedio de edad. Se realizó una técnica de muestreo no probabilístico intencional (Bisquerra, 2012) donde se garantizó que todos los practicantes que competían estaban en activo en el momento de recoger los datos. La participación fue anónima y consentida.

Tabla 1. Número y edad de los grupos participantes.

\begin{tabular}{cccccc}
\hline DEPORTES & GRUPOS POR NIVEL & $n$ & $\mathrm{M}$ & $\mathrm{DT}$ & $\%$ \\
\hline Boxeo & Practicante regular & 62 & 26,5 & 6,68 & $23 \%$ \\
$(n=108)$ & Competidor Regional & 52 & 25,8 & 5,49 & $19,3 \%$ \\
Kick- & Competidor Nacional & 54 & 28,2 & 4,44 & $20,1 \%$ \\
boxing & Competidor Internacional & 47 & 28,5 & 5,53 & $17,5 \%$ \\
$(n=107)$ & No practicante & 54 & 23,8 & 6,23 & $20,1 \%$ \\
& Total muestra & 269 & 26,5 & 5,97 & $100 \%$ \\
\hline
\end{tabular}

Notas: $\mathrm{n}=$ tamaño muestra, $\mathrm{M}=$ =media aritmética, $\mathrm{DT}=$ desviación típica, \%=porcentaje; las medias están referidas a su promedio de edad. 


\section{Instrumentos}

Cuestionario sociodemográfico

Se elaboró con el objetivo de caracterizar a los sujetos de la investigación respecto a variables como la edad, género, disciplina y nivel deportivo, así como años, días y horas semanales de práctica.

Inventario de la Expresión de la Ira Estado-Rasgo (STAXI-2)

Se utilizó el Inventario de la Expresión de la Ira Estado-Rasgo (STAXI-2) de Spielberger (1999) validado al contexto español por Miguel-Tobal et al. (2001). Está compuesto por 49 reactivos divididos en 6 escalas: Estado de Ira 15 ítems (p.e.: "Estoy cabreado"), Rasgo de Ira 10 ítems (p.e.: "Me caliento rápidamente"), Expresión Externa de la Ira 6 ítems (p.e.: "Expreso mi ira"), Expresión Interna de la Ira 6 ítems (p.e.: "Me guardo para mí lo que siento"), Control Externo de la Ira 6 ítems (p.e.: "Controlo mi temperamento") y Control Interno de la Ira 6 ítems (p.e.: "Respiro profundamente y me relajo"). La escala estado de ira está formada por 3 subescalas: Sentimiento de la Ira 5 ítems (p.e.: "Estoy enfadado"), Expresión Verbal de la Ira 5 ítems (p.e.: "Me gustaría decir tacos") Expresión Física de la Ira 5 ítems (p.e.: "Le pegaría a alguien”), mientras que la escala Rasgo de Ira está constituida por 2 subescalas: Temperamento de Ira 5 ítems (p.e.: "Tengo un carácter irritable") y Reacción a la Ira 5 ítems (p.e.: "Me enfado cuando se me trata injustamente"). El cuestionario se puntúa con una escala Likert de 4 puntos desde 1 (no, en absoluto) a 4 (mucho). La escala estado de ira evalúa intensidad, mientras que el resto de escalas miden frecuencia. A partir de las escalas de expresión y control de la ira se puede extraer el Índice de Expresión de Ira que ofrece una medida general para la expresión y control de la ira. La autores reportaron una correlación test-retest de ,71 y un coeficiente alpha de Cronbach de ,89 para la escala Estado de Ira, de ,82 para Rasgo de Ira, y de ,69 para Expresión de Ira (Miguel-Tobal et al., 2001). En el presente estudio, la escala STAXI-2 obtuvo un índice de fiabilidad global de ,85 y por escalas y subescalas los valores fueron: Estado de Ira ,87, Rasgo de Ira ,81, Expresión Externa de Ira ,63, Expresión Interna de Ira ,64, Control Interno de Ira ,82, Control Externo de Ira ,77, Sentimiento de Ira ,79, Expresión Física de la Ira ,75, Expresión Verbal de la Ira ,73, Temperamento de Ira ,83, Reacción a la Ira,72 e Indice de Expresión de Ira ,69. Los índices de fiabilidad inferiores a ,70 son considerados aceptables ya que el número de ítems es reducido (Nunally y Bernstein, 1994).

\section{Procedimiento}

En primer lugar se obtuvo el permiso del Comité de Ética de la Universidad de los investigadores previo al inicio de la toma de datos. Posteriormente, en el caso de los participantes deportistas, se contactó con los entrenadores y los propios practicantes para explicarles el objetivo del estudio. Tras su aprobación, los cuestionarios fueron cumplimentados por los deportistas en el propio gimnasio al finalizar una sesión de entrenamiento y supervisados por un investigador experimentado. En el caso de los no deportistas, se contactó con personas no practicantes del entorno de los investigadores. La duración aproximada para cubrir los cuestionarios en todos los casos fue de unos 20 minutos. Se instó a los participantes a que respondieran con la mayor sinceridad posible y se les informó de que sus datos serían tratados confidencialmente. Respecto a las preguntas del cuestionario, se aclaró a los participantes practicantes que los reactivos estaban referidos a la vida diaria y no a la competición o práctica deportiva.

\section{Análisis de datos}

Los datos fueron tratados con el programa SPSS en su versión 22 (IBM, Chicago, IL). Como se verá en la siguiente anotación, ha sido un error nuestro pues los alpha de Cronbach los incluimos en el apartado anterior de "instrumentos" y no aquí. Mejor eliminar lo coloreado para que quede: “...(IBM, Chicago, IL). Se calcularon las medias, desviaciones típicas, mínimos, máximos, asimetría y curtosis como descriptivos generales de los participantes. Para la comparación entre grupos y dada la distribución no normal de las variables tras realizar el test de Kolmogorov-Smirnov $(p<, 05)$, se realizó la prueba no paramétrica de contraste de medias $\mathrm{H}$ de Kruskal-Wallis $(p<, 05)$. Posteriormente, y con el objeto de conocer entre qué grupos había diferencias estadísticamente significativas, se realizó la prueba post hoc T2 de Tamhane. Asimismo, se ejecutó la prueba Kruskal-Wallis $(p<, 05)$ previo a utilizar la prueba U MannWhitney $(p<, 05)$ para conocer las posibles diferencias en función del tipo de deporte. A continuación, y para determinar la correlación entre las dimensiones de la ira, la edad, los años de práctica, los días y las horas semanales de entrenamiento, se realizó la prueba de correlaciones Rho de Spearman.

\section{Resultados}

\section{Análisis descriptivos}

En la tabla 2 se presentan las medias, desviaciones típicas, mínimos, máximos, asimetría y curtosis de las diferentes dimensiones de la ira. Los participantes de este estudio muestran medias más altas en el índice de expresión de ira y más bajas en la subescala de expresión física de la ira. Todas las dimensiones de la ira muestran niveles de asimetría y curtosis adecuados (entre $-2 y+2$ ) exceptuando la subescala expresión física de la ira en la que la curtosis se eleva por encima de los valores descritos. 
Tabla 2. Estadísticos descriptivos.

\begin{tabular}{|c|c|c|c|c|c|c|}
\hline Variables & $\mathrm{M}$ & DT & MIN & MAX & ASIMETRIA & CURTOSIS \\
\hline Estado de Ira & 23,05 & 5,96 & 15 & 49 & 99 & 1,45 \\
\hline Rasgo de Ira & 19,56 & 5,10 & 10 & 35 &, 52 &, 06 \\
\hline Expresión Externa de Ira & 10,27 & 2,74 & 6 & 19 &, 55 &,- 02 \\
\hline Expresión Interna de Ira & 12,17 & 3,20 & 6 & 22 &, 37 &,- 38 \\
\hline Control Interno de Ira & 14,25 & 4,17 & 6 & 24 &, 26 &,- 40 \\
\hline Control Externo de Ira & 18,15 & 4,46 & 6 & 24 &,- 56 &,- 51 \\
\hline Sentimiento de Ira & 7,92 & 2,43 & 5 & 19 &, 85 &, 89 \\
\hline Expresión Física de la Ira & 6,60 & 1,99 & 5 & 16 & 1,82 & 3,89 \\
\hline Expresión Verbal de la Ira & 8,52 & 2,67 & 5 & 16 & ,62 &,- 27 \\
\hline Temperamento de Ira & 7,83 & 2,83 & 5 & 19 & 1,10 & ,91 \\
\hline Reacción a la Ira & 11,72 & 3,22 & 5 & 20 &, 23 &,- 42 \\
\hline Índice Expresión Ira & 26,04 & 8,77 & 6 & 52 & ,26 &,- 28 \\
\hline
\end{tabular}

Notas: $M=$ media aritmética, $D T=$ desviación típica, $M I N=$ mínimos, $M A X=$ máximos.

\section{Análisis de contraste}

Para conocer si existían diferencias significativas en las variables de estudio en función de los grupos se realizó la prueba no paramétrica de contraste de medias $\mathrm{H}$ de Kruskal-Wallis $(p<, 05)$. Los resultados muestran la existencia de diferencias significativas en todas las variables objeto de estudio menos en la expresión y control interno de ira (ver tabla 3).

Tabla 3. Medias, desviaciones típicas y prueba H de Kruskal-Wallis por grupos.

\begin{tabular}{|c|c|c|c|c|c|c|}
\hline Variables & $\begin{array}{c}\text { No practicantes } \\
\text { M (DE) }\end{array}$ & $\begin{array}{l}\text { Practicantes } \\
\text { regulares } \\
\text { M (DE) }\end{array}$ & $\begin{array}{c}\text { Competidores } \\
\text { Regionales } \\
\text { M (DE) }\end{array}$ & $\begin{array}{c}\text { Competidores } \\
\text { Nacionales } \\
\text { M (DE) }\end{array}$ & $\begin{array}{c}\text { Competidores } \\
\text { Internacionales } \\
\text { M (DE) }\end{array}$ & $\begin{array}{c}\text { Valores } \\
\quad p\end{array}$ \\
\hline Años de práctica & 0 & $3,73(3,72)$ & $5,50(4,56)$ & $8,89(5,36)$ & $13,11(6,13)$ &, 00 \\
\hline Días semanales de práctica & 0 & $3,32(1,00)$ & $4,37(1,10)$ & $5,04(, 97)$ & $5,60(, 64)$ &, 00 \\
\hline Horas semanales de práctica & 0 & $4,69(2,39)$ & $7,35(3,95)$ & $11,52(4,12)$ & $17,81(6,53)$ &, 00 \\
\hline Estado de Ira & $24,70(6,02)$ & $25,30(6,56)$ & $23,48(5,64)$ & $21,68(5,13)$ & $19,29(3,98)$ &, 00 \\
\hline Rasgo de Ira & $20,83(4,68)$ & $20,48(5,17)$ & $19,63(5,75)$ & $18,79(4,87)$ & $17,68(4,40)$ &, 00 \\
\hline Expresión Externa de Ira & $11,33(2,94)$ & $10,82(2,63)$ & $10,57(2,62)$ & $9,68(2,56)$ & $8,70(2,14)$ &, 00 \\
\hline Expresión Interna de Ira & $12,87(3,33)$ & $12,20(3,02)$ & $11,98(3,08)$ & $12,57(3,52)$ & $11,06(2,82)$ & ,08 \\
\hline Control Interno de Ira & $13,80(4,47)$ & $14,95(4,16)$ & $14,03(3,66)$ & $13,90(4,22)$ & $14,44(4,32)$ &, 45 \\
\hline Control Externo de Ira & $17,50(4,56)$ & $16,82(4,81)$ & $18,46(3,96)$ & $18,77(3,92)$ & $19,59(4,55)$ & 01 \\
\hline Sentimiento de Ira & $8,59(2,44)$ & $8,74(2,75)$ & $7,75(2,09)$ & $7,51(2,22)$ & $6,76(2,00)$ &, 00 \\
\hline Expresión Física de la Ira & $6,85(2,25)$ & $7,24(2,38)$ & $6,80(2,01)$ & $6,14(1,53)$ & $5,78(, 99)$ &, 00 \\
\hline Expresión Verbal de la Ira & $9,25(2,65)$ & $9,32(2,54)$ & $8,92(2,60)$ & $8,01(2,71)$ & $6,74(1,97)$ &, 00 \\
\hline Temperamento de Ira & $8,16(2,89)$ & $8,51(3,00)$ & $8,09(2,97)$ & $7,35(2,63)$ & $6,82(2,30)$ &, 00 \\
\hline Reacción a la Ira & $12,66(2,85)$ & $11,96(3,20)$ & $11,53(3,46)$ & $11,44(3,48)$ & $10,85(2,88)$ &, 03 \\
\hline Índice Expresión Ira & $28,87(9,22)$ & $27,25(9,02)$ & $26,05(8,31)$ & $25,57(8,42)$ & $21,72(7,30)$ &, 00 \\
\hline
\end{tabular}

Notas: $p<, 05 ; M=$ media aritmética, $D T=$ desviación típica, $p=$ valor de significación.

Posteriormente, se realizó la prueba post hoc T2 de Tamhane para conocer entre qué grupos había diferencias estadísticamente significativas (ver tabla 4). Se observa que las diferencias siempre se producen entre los competidores internacionales y los grupos de menor nivel, especialmente entre los internacionales y los practicantes regulares y los no practicantes. Solo en las escalas estado y expresión externa de ira y en la subescala expresión física de ira los nacionales también presentaron diferencias significativas con otros grupos de menor nivel. 
Tabla 4. Prueba post hoc T2 de Tamhane.

\begin{tabular}{|c|c|c|c|}
\hline Variables & Grupos & Diferencia de medias (I-J) & Valores $p$ \\
\hline \multirow[t]{4}{*}{ Estado de Ira } & Internacional - regional & $-4,18290^{*}$ & ,00 \\
\hline & Internacional - practicante regular & $-6,00858^{*}$ & 00 \\
\hline & Internacional - no practicante & $-5,40583^{*}$ &, 00 \\
\hline & Nacional - practicante regular & $-3,62127^{*}$ &, 01 \\
\hline \multirow[t]{2}{*}{ Rasgo de Ira } & Internacional - practicante regular & $-2,80302^{*}$ & ,02 \\
\hline & Internacional - no practicante & $-3,15248^{*}$ &, 00 \\
\hline \multirow[t]{4}{*}{ Expresión Externa de Ira } & Internacional - regional & $-1,87480^{*}$ &, 00 \\
\hline & Internacional - practicante regular & $-2,12045^{*}$ & 00 \\
\hline & Internacional - no practicante & $-2,63121^{*}$ &, 00 \\
\hline & Nacional - no practicante & $-1,64815^{*}$ &, 00 \\
\hline Control Externo de Ira & Internacional - practicante regular & $-2,77316^{*}$ &, 02 \\
\hline \multirow[t]{2}{*}{ Sentimiento de Ira } & Internacional - practicante regular & $-1,97598^{*}$ & 00 \\
\hline & Internacional - no practicante & $-1,82664^{*}$ & 00 \\
\hline \multirow[t]{4}{*}{ Expresión Física de la Ira } & Internacional - practicante regular & $-1,45470^{*}$ &, 00 \\
\hline & Internacional - regional & $-1,02046^{*}$ &, 01 \\
\hline & Internacional - no practicante & $-1,06462^{*}$ &, 02 \\
\hline & Nacional - practicante regular & $-1,09379^{*}$ &, 03 \\
\hline \multirow[t]{3}{*}{ Expresión Verbal de la Ira } & Internacional - regional & $-2,17840^{*}$ &, 00 \\
\hline & Internacional - practicante regular & $-2,57790^{*}$ & 00 \\
\hline & Internacional - no practicante & $-2,51458^{*}$ & 00 \\
\hline Temperamento de Ira & Internacional - practicante regular & $-1,16428^{*}$ &, 01 \\
\hline Reacción a la Ira & Internacional - no practicante & $-1,81560^{*}$ & ,02 \\
\hline \multirow[t]{2}{*}{ Índice de Expresión de Ira } & Internacional - practicante regular & $-5,53466^{*}$ & 00 \\
\hline & Internacional - no practicante & $-7,14697^{*}$ &, 00 \\
\hline
\end{tabular}

Tabla 5. Medias, desviaciones típicas y prueba U Mann-Whitney en función del deporte.

\begin{tabular}{|c|c|c|c|c|}
\hline Variables & $\begin{array}{c}\text { Boxeo } \\
\text { M (DT) }\end{array}$ & $\begin{array}{c}\text { Kickboxing } \\
\text { M (DT) }\end{array}$ & Z & $\begin{array}{c}\text { Valores } \\
p \\
\end{array}$ \\
\hline Estado de Ira & $22,70(5,56)$ & $22,57(6,24)$ &,- 53 &, 59 \\
\hline Rasgo de Ira & $19,77(4,80)$ & $18,70(5,47)$ & $-1,94$ &, 05 \\
\hline Expresión Externa de Ira & $10,14(2,72)$ & $9,87(2,53)$ &,- 66 &, 50 \\
\hline Expresión Interna de Ira & $12,12(2,95)$ & $11,76(3,34)$ & $-1,03$ & ,29 \\
\hline Control Interno de Ira & $14,62(4,25)$ & $14,09(3,93)$ &,- 93 & ,35 \\
\hline Control Externo de Ira & $18,27(4,25)$ & $18,35(4,63)$ &,- 38 & ,70 \\
\hline Sentimiento de Ira & $7,89(2,17)$ & $7,62(2,62)$ & $-1,43$ & ,15 \\
\hline Expresión Física de la Ira & $6,59(1,78)$ & $6,49(2,06)$ &,- 97 &, 50 \\
\hline Expresión Verbal de la Ira & $8,21(2,64)$ & $8,45(2,67)$ &,- 67 & ,33 \\
\hline Temperamento de Ira & $8,01(2,72)$ & $7,48(2,90)$ & $-1,94$ & ,05 \\
\hline Reacción a la Ira & $11,75(3,12)$ & $11,21(3,42)$ & $-1,27$ &, 20 \\
\hline Índice Expresión Iira & $25,47(8,39)$ & $25,19(8,71)$ &,- 09 & 92 \\
\hline
\end{tabular}

Notas: $p<, 05 ; M=$ media aritmética, $D T=$ desviación típica, $Z=$ contraste de KolmogorovSmirnov. $p=$ valor de significación.
Con el fin de determinar si había diferencias significativas en función del deporte se realizó, en primer lugar, la prueba no paramétrica de contraste de medias $\mathrm{H}$ de Kruskal-Wallis $(p<, 05)$ y posteriormente la prueba no paramétrica U MannWhitney $(p<, 05)$ (ver tabla 5$)$. Los resultados muestran la no existencia de diferencias significativas entre los diferentes componentes de la ira en función del deporte.

\section{Análisis correlacional}

Para determinar la correlación entre los diferentes componentes de la ira y la edad, los años de práctica, los días y horas semanales de entrenamiento de los participantes se realizó la prueba de correlaciones Rho de Spearman (ver tabla 6). Se observa una correlación significativa negativa entre la edad y el rasgo de ira (subescalas temperamento y reacción a la ira) y la expresión externa. Los años de práctica correlacionan significativamente (positiva y negativamente según el caso) con todos los componentes de la ira, exceptuando con el control interno. Los días semanales de entrenamiento correlacionan 
negativamente de manera significativa con el estado de ira (subescalas sentimiento y expresión verbal de la ira) y la expresión externa de ira. Finalmente, las horas semanales de práctica correlacionan significativamente (positiva y negativamente según el caso) con todas las escalas y subescalas del inventario exceptuando con el control interno de ira.

\section{Discusión}

El objetivo de este estudio fue analizar si existían diferencias en los niveles de ira en función de la disciplina y el nivel deportivo en una muestra de deportistas de contacto pleno y un grupo de no practicantes y determinar las posibles correlacio- nes existentes entre la ira y la edad, los años, los días y las horas semanales de práctica. Los resultados obtenidos muestran que los deportistas internacionales presentan niveles significativamente más bajos de ira que los practicantes regulares y que los no practicantes de actividad física. Asimismo, se han establecido correlaciones significativas entre la edad, los ańos, los días y las horas de práctica y diferentes dimensiones de la ira. No se han encontrado diferencias significativas en el nivel de ira entre los practicantes regulares de estas disciplinas y los no practicantes de actividad física. Finalmente, tampoco se han encontrado diferencias significativas en los niveles de ira entre los practicantes de boxeo y los de kickboxing.

Tabla 6. Correlaciones Rho de Spearman.

\begin{tabular}{|c|c|c|c|c|c|c|c|c|c|c|c|c|c|c|c|c|c|}
\hline Factores & & 1 & 2 & 3 & 4 & 5 & 6 & 7 & 8 & 9 & 10 & 11 & 12 & 13 & 14 & 15 & 16 \\
\hline 1. Edad & $\begin{array}{l}\text { C.C. } \\
p\end{array}$ & --- & & & & & & & & & & & & & & & \\
\hline 2. Años de práctica & $\begin{array}{l}\text { C.C. } \\
p\end{array}$ & $\begin{array}{l}, 51^{* *} \\
, 00\end{array}$ & -- & & & & & & & & & & & & & & \\
\hline 3. Días sem. de práctica & $\begin{array}{l}\text { C.C. } \\
p\end{array}$ & $\begin{array}{l}, 13^{*} \\
, 02\end{array}$ & $\begin{array}{l}, 68^{* *} \\
, 00\end{array}$ & -.- & & & & & & & & & & & & & \\
\hline 4. Horas sem. de práctica & $\begin{array}{l}\text { C.C. } \\
p\end{array}$ & $\begin{array}{l}, 21^{* *} \\
, 00\end{array}$ & $\begin{array}{l}, 75^{* *} \\
, 00\end{array}$ & $\begin{array}{l}, 90^{* *} \\
, 00\end{array}$ & -- & & & & & & & & & & & & \\
\hline 5. Estado de ira & $\begin{array}{l}\text { C.C. } \\
p\end{array}$ & $\begin{array}{l}-, 08 \\
, 17\end{array}$ & $\begin{array}{l}-, 31^{* *} \\
, 00\end{array}$ & $\begin{array}{l}{ }^{k}-, 20^{* *} \\
, 00\end{array}$ & $\begin{array}{l}{ }^{*}-, 27^{* *} \\
, 00\end{array}$ & -.- & & & & & & & & & & & \\
\hline 6. Rasgo de ira & $\begin{array}{l}\text { C.C. } \\
p\end{array}$ & $\begin{array}{l}-, 19^{* *} \\
, 00\end{array}$ & $\begin{array}{l}-, 31^{* *} \\
, 00\end{array}$ & $\begin{array}{l}{ }^{k}-, 11 \\
, 06\end{array}$ & $\begin{array}{l}-, 16^{* *} \\
, 00\end{array}$ & $\begin{array}{l}, 53^{* *} \\
, 00\end{array}$ & --- & & & & & & & & & & \\
\hline 7. Expresión externa de ira & $\begin{array}{l}\text { C.C. } \\
p\end{array}$ & $\begin{array}{l}-, 18^{* *} \\
, 00\end{array}$ & $\begin{array}{l}-, 33^{* *} \\
, 00\end{array}$ & $\begin{array}{l}{ }^{*}-, 19^{* *} \\
, 00\end{array}$ & $\begin{array}{l}-, 23^{* *} \\
, 00\end{array}$ & $\begin{array}{l}, 59^{* *} \\
, 00\end{array}$ & $\begin{array}{l}.56^{* *} \\
.00\end{array}$ & --. & & & & & & & & & \\
\hline 8. Expresión interna de ira & $\begin{array}{l}\text { C.C. } \\
p\end{array}$ & $\begin{array}{l}-, 05 \\
, 41\end{array}$ & $\begin{array}{l}-, 17^{* *} \\
, 00\end{array}$ & $\begin{array}{l}{ }^{*}-, 09 \\
, 10\end{array}$ & $\begin{array}{l}-, 13^{*} \\
, 03\end{array}$ & $\begin{array}{l}, 36^{* *} \\
, 00\end{array}$ & $\begin{array}{l}, 49^{* *} \\
, 00\end{array}$ & $\begin{array}{l}, 25^{* *} \\
, 00\end{array}$ & -- & & & & & & & & \\
\hline 9. Control interno de ira & $\begin{array}{l}\text { C.C. } \\
p\end{array}$ & $\begin{array}{l}, 02 \\
, 71\end{array}$ & $\begin{array}{l}-, 02 \\
, 71\end{array}$ & $\begin{array}{l}-, 03 \\
, 61\end{array}$ & $\begin{array}{l}-, 03 \\
, 61\end{array}$ & $\begin{array}{l}-, 04 \\
, 51\end{array}$ & $\begin{array}{l}, 01 \\
, 75\end{array}$ & $\begin{array}{l}-, 07 \\
, 23\end{array}$ & $\begin{array}{l}, 13^{*} \\
, 03\end{array}$ & --- & & & & & & & \\
\hline 10. Control externo de ira & $\begin{array}{l}\text { C.C. } \\
p\end{array}$ & $\begin{array}{l}, 02 \\
, 72\end{array}$ & $\begin{array}{l}, 18^{* *} \\
, 00\end{array}$ & $\begin{array}{l}, 11 \\
, 05\end{array}$ & $\begin{array}{l}, 15^{*} \\
, 01\end{array}$ & $\begin{array}{l}-, 37^{* *} \\
, 00\end{array}$ & $\begin{array}{l}{ }^{*}-, 36^{*} \\
, 00\end{array}$ & $\begin{array}{l}-, 41^{* *} \\
, 00\end{array}$ & $\begin{array}{l}-, 02 \\
, 68\end{array}$ & $\begin{array}{l}, 24^{* *} \\
, 00\end{array}$ & --- & & & & & & \\
\hline 11. Sentimiento de ira & $\begin{array}{l}\text { C.C. } \\
p\end{array}$ & $\begin{array}{l}-, 11 \\
, 06\end{array}$ & $\begin{array}{l}-, 28^{* *} \\
, 00\end{array}$ & $\begin{array}{c}*-, 15^{*} \\
, 01\end{array}$ & $\begin{array}{l}-, 22^{* *} \\
, 00\end{array}$ & $\begin{array}{l}, 86^{* *} \\
, 00\end{array}$ & $\begin{array}{l}, 52^{* *} \\
, 00\end{array}$ & $\begin{array}{l}, 49^{* *} \\
, 00\end{array}$ & $\begin{array}{l}-, 40^{* *} \\
, 00\end{array}$ & $\begin{array}{l}-, 03 \\
, 55\end{array}$ & $\begin{array}{l}-, 29^{* *} \\
, 00\end{array}$ & *-. & & & & & \\
\hline 12. Expresión física de la ira & $\begin{array}{l}\text { C.C. } \\
p\end{array}$ & $\begin{array}{l}-, 10 \\
, 08\end{array}$ & $\begin{array}{l}-, 18^{* *} \\
, 00\end{array}$ & $\begin{array}{l}{ }^{*}-, 11 \\
, 05\end{array}$ & $\begin{array}{l}-, 15^{*} \\
, 01\end{array}$ & $\begin{array}{l}, 74^{* *} \\
, 00\end{array}$ & $\begin{array}{l}, 43^{*} \\
, 00\end{array}$ & $\begin{array}{l}, 39^{* *} \\
, 00\end{array}$ & $\begin{array}{l}, 27^{* *} \\
, 00\end{array}$ & $\begin{array}{l}-, 01 \\
, 85\end{array}$ & $\begin{array}{l}-, 34^{* *} \\
, 00\end{array}$ & $\begin{array}{l}{ }^{k}, 56^{* *} \\
, 00\end{array}$ & '.- & & & & \\
\hline 13. Expresión verbal de la ira & $\begin{array}{l}\text { C.C. } \\
p\end{array}$ & $\begin{array}{l}, 01 \\
, 81\end{array}$ & $\begin{array}{l}-, 28^{* *} \\
, 00\end{array}$ & $\begin{array}{l}*_{-}, 22^{* *} \\
, 00\end{array}$ & $\begin{array}{l}{ }^{*}-, 29^{* *} \\
, 00\end{array}$ & $\begin{array}{l}, 84^{* *} \\
, 00\end{array}$ & $\begin{array}{l}, 36^{* *} \\
, 00\end{array}$ & $\begin{array}{l}, 53^{* *} \\
, 00\end{array}$ & $\begin{array}{l}, 22^{* *} \\
, 00\end{array}$ & $\begin{array}{l}-, 01 \\
.85\end{array}$ & $\begin{array}{l}-, 28^{* *} \\
, 00\end{array}$ & $\begin{array}{l}{ }^{*}, 57^{* *} \\
, 00\end{array}$ & $\begin{array}{l}, 48^{* *} \\
, 00\end{array}$ & -- & & & \\
\hline 14. Temperamento de ira & $\begin{array}{l}\text { C.C. } \\
p\end{array}$ & $\begin{array}{l}-, 12^{*} \\
, 04\end{array}$ & $\begin{array}{l}-, 27^{* *} \\
, 00\end{array}$ & $\begin{array}{l}{ }^{*}-, 11 \\
, 05\end{array}$ & $\begin{array}{l}-, 17^{* *} \\
, 00\end{array}$ & $\begin{array}{l}, 63^{* *} \\
, 00\end{array}$ & $\begin{array}{l}, 78^{* *} \\
, 00\end{array}$ & $\begin{array}{l}, 63^{* *} \\
, 00\end{array}$ & $\begin{array}{l}, 39^{* *} \\
, 00\end{array}$ & $\begin{array}{l}-.07 \\
.20\end{array}$ & $\begin{array}{l}-, 48^{* *} \\
, 00\end{array}$ & $\begin{array}{l}{ }^{k}, 51^{* *} \\
, 00\end{array}$ & $\begin{array}{l}, 45^{* *} \\
, 00\end{array}$ & $\begin{array}{l}, 46^{* *} \\
, 00\end{array}$ & ... & & \\
\hline 15. Reacción a la ira & $\begin{array}{l}\text { C.C. } \\
p\end{array}$ & $\begin{array}{l}-, 20^{* *} \\
, 00\end{array}$ & $\begin{array}{c}{ }^{*}-, 28^{* *} \\
00\end{array}$ & $\begin{array}{c}{ }^{*}, 09 \\
, 10\end{array}$ & $\begin{array}{l}-, 13^{*} \\
, 03\end{array}$ & $\begin{array}{l}, 31^{* *} \\
, 00\end{array}$ & $\begin{array}{l}, 87^{* *} \\
, 00\end{array}$ & $\begin{array}{l}, 37^{* *} \\
, 00\end{array}$ & $\begin{array}{l}, 43^{* *} \\
00\end{array}$ & $\begin{array}{l}.08 \\
.15\end{array}$ & $\begin{array}{l}-, 19^{* *} \\
, 00\end{array}$ & $\begin{array}{l}, 32^{* *} \\
00\end{array}$ & $\begin{array}{l}29^{* *} \\
, 00\end{array}$ & $\begin{array}{l}, 19^{* *} \\
, 00\end{array}$ & $\begin{array}{l}, 41^{* *} \\
, 00\end{array}$ & - & \\
\hline 16. Índice de expresión de ira & $\begin{array}{l}\text { C.C. } \\
p\end{array}$ & $\begin{array}{l}-, 10 \\
, 09 \\
\end{array}$ & $\begin{array}{l}-, 25^{* *} \\
, 00\end{array}$ & $\begin{array}{c}*-15^{*} \\
, 01 \\
\end{array}$ & $\begin{array}{l}-, 19^{* *} \\
, 00\end{array}$ & $\begin{array}{l}, 50^{* *} \\
, 00 \\
\end{array}$ & $\begin{array}{l}, 52^{* *} \\
, 00 \\
\end{array}$ & $\begin{array}{l}, 62 \\
, 00 \\
\end{array}$ & $\begin{array}{l}, 38^{* *} \\
, 00 \\
\end{array}$ & $\begin{array}{l}-.56^{* *} \\
.00\end{array}$ & $\begin{array}{l}, 75^{* *} \\
, 00 \\
\end{array}$ & $\begin{array}{l}, 45^{* *} \\
, 00 \\
\end{array}$ & $\begin{array}{l}, 39^{* *} \\
, 00\end{array}$ & $\begin{array}{l}, 38^{* *} \\
, 00\end{array}$ & $\begin{array}{l}, 59^{* *} \\
, 00\end{array}$ & $\begin{array}{l}, 34^{* *} \\
, 00\end{array}$ & --- \\
\hline
\end{tabular}

Notas: ${ }^{*} p<, 05{ }^{* *} p<, 01 ; C . C .=$ coeficiente de correlación, $p=$ valor de significación.

Respecto a la primera hipótesis, se encontró que hubo diferencias significativas entre los internacionales y los practicantes regulares en las escalas de estado, rasgo, expresión externa y control externo de ira, así como en todas las subescalas del inventario, exceptuando la reacción a la ira. Los competidores de nivel internacional presentaban niveles más bajos que 
los practicantes regulares. Asimismo, se encontró que también hubo diferencias significativas entre los internacionales y los no practicantes en las escalas de estado, rasgo y expresión externa y en las subescalas de sentimiento, expresión verbal, física y reacción a la ira. Como en el caso anterior, los competidores internacionales presentaban niveles más bajos. Estos datos revelan que el nivel deportivo es un elemento clave a tener en cuenta en el estudio de la ira en deportes de contacto pleno. Estos resultados están en consonancia con los encontrados por Maxwell, Visek y Moores (2009) en jugadores de baloncesto, de fútbol y de squash, los cuales mostraban que los deportistas de mayor nivel y más experimentados de estos deportes mostraban menores niveles de ira; la excepción provenía de los jugadores de rugby que no seguían esta misma tendencia

No se conocen estudios que hayan analizado el nivel de ira en practicantes de boxeo y kickboxing, pero los datos arrojados por Robazza, Bertollo y Bortoli (2006) con otro tipo de deportistas de contacto (judo, lucha libre y lucha grecoromana) revelaron la no existencia de diferencias significativas respecto a la ira en función del nivel de los participantes. Estos resultados van en contra de los expuestos en el presente trabajo, sin embargo, cabe destacar que tanto en el estudio de Robazza et al. (2006) como en la gran mayoría de los trabajos que han tratado de estudiar la ira multidimensionalmente en otras AAMM y DDCC (Oliva y Hernández, 2009; Oliva, Calleja y Hernández, 2011, Ruiz y Hanin, 2004b, 2011) los reactivos estaban referidos a la competición y no a la vida diaria, por lo que los resultados podrían haber sido diferentes de haber enfocado las preguntas a otros contextos de la vida diaria.

Las causas por las que los competidores internacionales presentaron menores niveles de ira en comparación con los grupos de nivel inferiores quizás puedan explorarse a partir de la segunda hipótesis planteada en este trabajo. Por una parte hay una correlación significativa entre todas las escalas y subescalas de la ira, exceptuando el control interno, y los años de práctica del deporte. Cuanto mayor es el nivel del deportista, más ańos lleva practicando la modalidad y más horas y días semanales entrena. La edad solo se correlacionó con la expresión externa y el rasgo (subescalas de temperamento y reacción a la ira) por lo que se puede entender que los ańos de práctica de estas disciplinas han contribuido, al menos, a reducir el estado de ira (sentimiento, expresión física y verbal de ira) y la expresión interna, así como a potenciar el control externo de los participantes, especialmente en el grupo de competidores internacionales que es el que más años de práctica tiene. En esta misma línea de argumentación, se observó que las horas semanales de entrenamiento correlacionaron de manera significativa con todas las escalas y subescalas de la ira, exceptuando el control interno. Quizá una de las causas por la que los internacionales tienen un per- fil iracundo significativamente mejor sea debido al efecto de catarsis que puede tener el deporte sobre esta variable, por lo que los competidores que durante más horas entrenan semanalmente están expuestos a un desgaste físico y psicológico que hace modificar su perfil iracundo. Maxwell et al. (2009) también plantean que a mayor número de tiempo de práctica deportiva (en su caso baloncesto, futbol y squash) menores niveles de ira. Los días semanales de entrenamiento solo correlacionaron significativamente con el estado (subescalas de sentimiento y expresión verbal) y la expresión externa de ira. Esto indica que son más decisivas las horas que un deportista entrena semanalmente que los días para observar diferencias en el perfil iracundo. Estos resultados coinciden con los aportados por otros autores (van Ingen, 2011) que hablan de la capacidad que tienen estas prácticas como forma de catarsis y canalización de la ira. Por otro lado, Maxwell et al. (2009) consideran que este fenómeno también se podría explicar en base al aprendizaje que realizan los deportistas veteranos, encontrando estrategias que les permiten manejar mejor sus emociones, disminuyendo su agresividad y su ira.

Respecto a la tercera hipótesis, no se encontraron diferencias entre los practicantes regulares y los no practicantes; lo que refleja que la práctica de estos deportes, a nivel regular, no hace aumentar los niveles de estado, rasgo y expresión de ira, ni reducir los de control de esta emoción, muy al contrario de la creencia popular existente. Estos datos concuerdan con otros aportados por Focht, Bouchard y Murphey (2000) en practicantes de otro tipo de AAMM y DDCC como judo y karate, que no presentaron diferencias significativas en las puntuaciones de ira total, instrumental, reactiva y control de ira en comparación con un grupo control de no practicantes. También concuerdan con los aportados por Ziaee et al. (2012) en los que los no practicantes de actividad física presentaban niveles significativamente superiores de ira que un grupo de nadadores.

Respecto a la cuarta hipótesis, no se encontraron diferencias significativas en las múltiples dimensiones de la ira estudiadas en relación al tipo de disciplina practicada. En otros estudios relacionados con las AAMM y DDCC como los de Boostani y Boostani (2012) y Sofia y Fernando (2012), sí que se encontraron diferencias en función del tipo de deporte practicado. No obstante, conviene aclarar que estas diferencias se produjeron entre deportes que no guardaron una fuerte relación entre sí; es decir, entre actividades sin contacto, con contacto controlado y con contacto pleno. Los resultados de nuestro estudio respecto a esta cuestión pueden deberse a un factor sociocultural del deporte. Por norma general, el kickboxing y el boxeo, aunque guardan ciertas diferencias técnico-tácticas, tal como afirman Vertonghen et al. (2014), atraen a un tipo de perfil poblacional muy similar, quizá por el hecho de que son actividades muy parecidas (deportes de ring y contacto pleno, utilizan una indumentaria parecida, se 
practican en los mismos lugares, los entrenadores en ocasiones son los mismos para una u otra disciplina, la sensación de desgaste físico y psicológico es similar...). Esto conduce a que tanto los practicantes regulares que se inician en estos deportes, como los propios competidores se muevan fluidamente entre estas disciplinas, practicando y compitiendo en ambas. Respecto a los niveles de ira encontrados en otros deportes, Maxwell et al. (2009) señalan que los jugadores de rugby encuestados presentaban los más altos y los de squash los más bajos, quedando los de fútbol y baloncesto en niveles intermedios.

A modo de conclusión podemos señalar que este trabajo ha abordado por primera vez el estudio de la ira desde un prisma multidimensional en un contexto como la vida diaria en deportes donde la bibliografía es casi inexistente. Los pocos trabajos realizados sobre estas modalidades no se han detenido a explorar variables determinantes en la práctica física como el nivel, la edad, los años de experiencia y los días y horas semanales de entrenamiento. Los resultados mostraron cómo el nivel, los años de práctica y las horas que un deportista entrena semanalmente son variables de especial relevancia en el estudio de la ira en deportes de contacto pleno. El tipo de disciplina practicada no parece haber afectado significativamente a esta variable, lo que indica la similitud que guardan estos deportes respecto a esta emoción.

Como limitaciones del estudio destacar, en primer lugar, la muestra limitada de cada uno de los grupos de análisis, lo que dificulta hacer generalizaciones. En segundo lugar, no se pudo controlar el momento en el que contestaron los cuestionarios los participantes del grupo de no practicantes. Hubiera sido interesante que todos ellos hubieran realizado el cuestionario en un momento concreto del día como se hizo con el grupo de deportistas (post-entrenamiento). Sin embargo, esto no se pudo realizar por cuestiones prácticas y por ende, los resultados podrían haberse modificado ligeramente.

Como futuras líneas de investigación sería interesante determinar las posibles diferencias en los niveles de ira dentro de las modalidades que constituyen el kickboxing, especialmente, haciendo distinción entre las de contacto pleno (al K.O.) y contacto controlado (sin K.O. intencional). Asimismo, consideramos que sería importante seguir aportando trabajos que exploraran la relación de esta variable con otras como la empatía o la violencia, no solo en el boxeo o el kickboxing, sino también en otras modalidades de contacto pleno que están en constante auge como el caso de las artes marciales mixtas (MMA- mixed martial arts) o el muay thai.

\section{Aplicaciones prácticas}

Los resultados obtenidos en este trabajo pueden ayudar a que se promueva la práctica de las modalidades de contacto pleno de kickboxing ya que no se ha visto que incrementen significativamente los niveles de estado, rasgo o expresión de ira, ni que reduzcan los de control de esta emoción, a pesar de las creencias populares existentes sobre estas disciplinas. Esta promoción, además, debería comenzar a darse desde la base y en diferentes ámbitos, tanto en el educativo como en el puramente deportivo o incluso extraescolar. Por ello, las implicaciones prácticas de este trabajo afectan tanto a los docentes como a los entrenadores y/o monitores encargados de enseñar estos deportes en los respectivos centros. El hecho de que no se hayan encontrado diferencias significativas en las diferentes escalas y subescalas del cuestionario entre practicantes y no practicantes hace viable la inclusión del kickboxing como un contenido válido para el contexto educativo. Esta implicación es de suma importancia para todos los docentes de Educación Física en aras de plantear la inclusión de nuevos contenidos curriculares de carácter innovador tradicionalmente no explotados en esta área, siempre desde la dimensión educativa. Esto podría ayudar a romper con prejuicios que afectan a deportes estereotipados y tradicionalmente relacionados con la agresividad, la violencia y el género masculino.

Por otra parte, los entrenadores deben ser conscientes de que el nivel deportivo afecta significativamente a muchas de las variables medidas en este estudio. Por lo tanto, parece necesario que estos y/o los deportistas, especialmente los competidores de nivel regional, recurran a profesionales de la psicología deportiva para aprender técnicas específicas y/o protocolos mentales. Esto podría ayudarles a gestionar adecuadamente variables como las analizadas en el presente estudio y conseguir un mayor nivel de rendimiento físico y mental en las competiciones.

\section{Referencias}

1. Adler, U.B. (2003). Karate and mental health: Can the practice of a martial art reduce aggressive tendencies? New York: ETD Collection for Pace University.

2. Ali, T., Emrah, A., Fahri, A., Necmettin, P. y Gülcan, T. (2010). Martial artists versus team sportsmen: aggressiveness and received social support. Ovidius University Annals, Series Physical Education and Sport/Science, Movement and Health, 10 (2), 202-207.

3. Ali, B.M., Ichraf, A., Khaled, T., Liwa, M. y Ali, E. (2013). Effect of gender and type of sport practiced on aggression and self-esteem in Tunisian athletes. Journal of Humanities and Social Science, 8 (4), 74-80.
4. Ato, M., López, J.J. y Benavente, A. (2013). Un sistema de clasificación de los diseños de investigación en psicología. Anales de Psicología, 29 (3), 1038-1059.

5. Berkowitz, L. (1996). Agresión: Causas, consecuencias y control. Bilbao, España: Editorial Desclée de Brouwer.

6. Bisquerra, R. (coord.) (2012). Metodología de la investigación educativa. Madrid: La Muralla.

7. Bjorkqvist, K. y Varhama, L. (2001). Attitudes toward violent conflict resolution among male and female karateka in comparison with practitioners of other sports. Perceptual and Motor Skills, 92 (2), 586-588. 
8. Bolgar, M., Janelle, C., y Giacobbi, P. (2008). Trait anger, appraisal, and coping differences among adolescent tennis players. Journal of Applied Sport Psychology, 20, 73-87.

9. Boostani, M.A. y Boostani, M.H. (2012). Investigation and comparing aggression in athletes in non-contact (swimming), limited contact (karate) and contactable (kickboxing) sport fields. Journal of Combat Sports and Martial Arts, 2 (2), 87-89.

10. van Bottenburg, M., Rijnen, B. y van Sterkenburg, J. (2005). Sports participation in the European Union. Trends and differences. Nieuwegein: W.J.H. Mulier Institute - Arko Sports Media.

11. Brown, D.R., Wang, Y., Ward, A., Ebbeling, C.B., Fortlage, L., Puleo, E., Benson, H. y Rippe, J.M. (1995). Chronic psychological effects of exercise and exercise plus cognitive strategies. Medicine and Science in Sports and Exercise, 27(5), 765-775.

12. Carrasco, M.A. y González, M.J. (2006). Aspectos conductuales de la agresión: definición y modelos explicativos. Acción Psicológica, 4 (2), 7-38.

13. Chiodo, S., Tessitore, A., Cortis, C., Cibelli, G., Lupo, C., Ammendolia, A., De Rosas, M. y Capranica, L. (2011). Stress-related hormonal and psychological changes to official youth Taekwondo competitions. European Journal of Sport Science, 12 (2), 113-120.

14. Daniels, K. y Thornton, E.W. (1990). An analysis of the relationship between hostility and training in the martial arts. Journal of Sport Science and Medicine, 8 (2), 95-101

15. Danish, S.J., Nellen, V.C., y Owens, S.S. (1996). Teaching life skills through sport: Community based programs for adolescents. En J. K. Van Raalte y B. W. Brewer (Eds.), Exploring sport and exercise psychology (pp. 205-225). Washington, DC: American Psychological Association.

16. Davenport, T. (2006). Perceptions of the contributions of the psychology to success in elite kickboxing. Journal of Sports Sciences and Medicine, 1 (5), 99-197.

17. Dunn, J.G.H., Gotwals, J.K., Causgrove, J., Dunn, J.C. y Syrotuik, D.G. (2006). Examining the relationship between perfectionism and trait anger in competitive sport. International Journal of Sport and Exercise Psychology, 4 (1), 7-24,

18. Egan, M.A. (1993). The effects of martial arts training on self-acceptance and anger reactivity with young adults. (Tesis doctoral no publicada). University of South California, South California.

19. Endresen, I.M. y Olweus, D. (2005). Participation in power sports and antisocial involvement in preadolescent and adolescent boys. Journal of Child Psychology and Psychiatry, 46 (5), 468-78.

20. Focht, B.C., Bouchard, L. J. y Murphey, M. (2000). Influence of martial arts training on the perception of experimentally induced pressure pain and selected psychological responses. Journal of Sport Behavior, 32 (3), 232-243.

21. Foster, Y. (1997). Brief aikido training versus karate and golf training and university students' scores on self-esteem, anxiety, and expression of anger. Perceptual and Motor Skills, 84 (2), 609-610.

22. van Ingen, C. (2011). Spatialities of anger: emotional geographies in a boxing program for survivors of violence. Sociology of Sport Journal, 28 (2), 171-188.

23. Loftian, S., Ziaee, V., Amini, H. y Mansournia, M. (2011). An analysis of anger in adolescent girls who practice the martial arts. International Journal of Pediatrics, 22 (1), 9.

24. Marks, M., Bukowska, K. y Biéc, P. (2012). Anxiety and emotional intelligence in members of the Poland national kickboxing team. Roz prawy Naukowe, 37, 31-38.

25. Maxwell, J. P., Visek, A. J. y Moores, E. (2009). Anger and perceived legitimacy of aggression in male Hong Kong Chinese athletes: Effects of type of sport and level of competition. Psychology of sport and exercise, 10 (2), 289-296

26. McDiarmid, A. (2007). The impact of traditional Taekwondo on self- control for middle school students. (Tesis doctoral no publicada). University of Denver, Denver.

27. McGowan, R.W. y Miller, M.J. (1989). Differences in mood states between successful and less successful karate participants. Perceptual and Motor Skills, 68, 505-506.

28. McGowan, R.W., Pierce, E.F. y Jordan, D. (1991). Mood alterations with a single bout of physical activity. Perceptual and Motor Skills, 72 (3-2), 1203-1209.

29. Miguel-Tobal, J., Casado, M., Can-Vindel, A. y Spielberger, C. D (2001). Inventario de Expresión de la Ira Estado-Rasgo STAXI-2. Madrid, Espańa: TEA Ediciones.

30. Nunnally, J. C., y Bernstein, I. H. (1994). Psychometric Theory. Nueva York: McGraw-Hill.

31. Oliva, F.J. y Calleja, N. (2010). Medición de la ira en el deporte de combate: validación del STAXI-2 en deportistas mexicanos. Revista Liberabit, 16 (1), 51-59.

32. Oliva, F.J. y Hernández, M.R. (2009). Efecto de la agresión colérica en el desempeńo deportivo en deportes de contacto: una revisión empírica. Journal of Behaviour, Health and Social Issues, 1 (1), 81-88.

33. Oliva, F.J., Calleja, N. y Hernández, M.R. (2011). La ira en el desempeño deportivo del judoka. Revista Latinoamericana de Medicina Conductual, 1 (2), 41-48.

34. Oliva, F.J., Calleja, N. y Hernández, M.R. (2012). Escala de creencias sobre la ira en el deporte de combate con atletas mexicanos. Revista Internacional de Medicina y Ciencias de la Actividad Física y el Deporte, 12 (45), 110-121.

35. Pieter, W. y Pieter, M.S. (2008). Mood and performance in aikido athletes. Acta Kinesiologiae Universitatis Tartuensis, 13, 107-116.

36. Proios, M. (2012). The relation between the emotion of "anger" and the cognitive processes in gymnastics athletes. International Quarterly of Sport Science, 1, 10-18.

37. Reynes, E. y Lorant, J. (2001). Do competitive martial arts attract aggressive children? Perceptual and Motor Skills, 93 (2), 382-386.

38. Reynes, E. y Lorant, J. (2002). Effect of traditional judo training on aggressiveness among young boys. Perceptual and Motor Skills, 94 (1) 21-25.

39. Reynes, E. y Lorant, J. (2004). Competitive martial arts and aggressiveness: a 2-yr. longitudinal study among young boys. Perceptual Motor and Skills, 98 (1), 103-115.

40. Robazza, C., Bertollo, M. y Bortoli, L. (2006). Frequency and direction of competitive anger in contact sports. Journal of Sports Medicine and Physical Fitness, 46, 501-508.

41. Ruiz, M. y Hanin, Y. (2003). Athletes' self perceptions of optimal states in karate: an application of the IZOF model. Revista de Psicología del Deporte, 13 (2), 229-224.

42. Ruiz, M. y Hanin, Y. (2004a). Metaphoric Description and Individualized Emotion Profiling of Performance States in Top Karate Athlete. Journal of Applied Sport Psychology, 16 (3), 258-273.

43. Ruiz, M. y Hanin, Y. (2004b). Idiosyncratic description of anger states in skilled Spanish karate athletes: an application of the IZOF model. Revista de Psicología del Deporte, 13 (1), $75-93$.

44. Ruiz, M. y Hanin, Y. (2011). Perceived impact of anger on performance of skilled karate athletes. Psychology of Sport and Exercise, 12 (3), 242-249.

45. Sofia, R. y Fernando, J. (2010). As relaçôes entre percepção de ameaça, ansiedade, raiva e agressividade no desporto: um estudo comparativo em modalidades de contacto. Revista Portuguesa de Ciências do Desporto, 13 (2), 44-56.

46. Spielberger C.D., Jacobs G.A., Russell, S. y Crane R.S. (1983). As sessment of anger: The state-trait anger scale. In: JN Butcher, C.D. Spielberger, (Eds.). Advances in Personality Assessment (vol. 2 pp. 159187). Hillsdale, NJ: Lawrence Erlbaum.

47. Spielberger, C.D., Johnson, E., Russell, S., Crane, R.S., Jacobs, G.A. y 
Worden, T. (1985). The experience and expression of anger: Construction and validation of an anger expression scale. In M. Chesney y R. Rosenman (Eds.). Anger and hostility in cardiovascular and behavioral disorders (pp. 5-30). Hemisphere: McGraw-Hill

48. Spielberger, C. D. (1999). Manual for the State-Trait Anger Expression Inventory, STAXI-2. Odessa, FL: Psychological Assessment Resource.

49. Steyn, B.J.M. y Roux, S. (2009). Aggression and psychological wellbeing of adolescent Tae Kwon Do participants in comparison with hockey participants and a non-sport group. African Journal for Physical, Health Education, Recreation and Dance, 15 (1), 32-43.

50. Szabo, A. y Parkin, A.M. (2001). The psychological impact of training deprivation in martial artists. Psychology of Sport and Exercise, 2 (3), 187-199.

51. Szabo, A. y Urbán, F. (2014). Do combat sports develop emotional intelligence? Kinesiology, 46 (1), 53-60.

52. Theeboom, M. y De Knop, P. (1999). Asian martial arts and ap- proaches of instruction in physical education. European Journal of Physical Education, 4, 146-161.

53. Vertonghen, J. y Theeboom, M. (2010). The social-psychological outcomes of martial arts practice among youth: a review. Journal of Sports Science and Medicine, 9, 528-537.

54. Vertonghen, J., Theeboom, M. y Pieter, W. (2014). Mediating factors in martial arts and combat sports: an analysis of the type of martial art, characteristics, and social background of young participants. Per ceptual and Motor Skills, 118 (1), 41-61.

55. WAKO (2012). Ring Sports General Rules. Recuperado de http://www. wakomexico.com/attachments/Chapter-2_English.pdf

56. Ziaee, V., Loftian, S., Amini, H., Mansournia, M. y Memari, A. (2012). Anger in adolescent boy athletes: a comparison among Judo, karate, swimming and non athletes. Iranian Journal of Pediatrics, 22 (1), 9-14. 